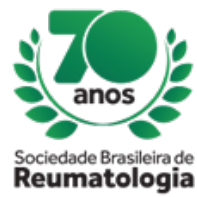

\title{
ASIA SYNDROME IN SLE PATIENT AFTER VACCINATION FOR HEPATITIS B
}

Raissa Velasques de Figueiredo (Universidade Federal de Ciências da Saúde de Porto Alegre, Porto Alegre, RS, Brasil), Maria Lúcia Lemos Lopes (Universidade Federal de Ciências da Saúde de Porto Alegre, Porto Alegre, RS, Brasil), Tatiana Freitas Tourinho (Universidade Federal de Ciências da Saúde de Porto Alegre, Porto Alegre, RS, Brasil), Maria Odete Esteves Hilário (Universidade Federal de Ciências da Saúde de Porto Alegre, Porto Alegre, RS, Brasil), Rodrigo Pereira Duquia (Universidade Federal de Ciências da Saúde de Porto Alegre, Porto Alegre, RS, Brasil), Gilberto Scanagatta (Universidade Federal de Ciências da Saúde de Porto Alegre, Porto Alegre, RS, Brasil), Rafael Coradin (Universidade Federal de Ciências da Saúde de Porto Alegre, Porto Alegre, RS, Brasil), Luana Ribeiro Carlos (Universidade Federal de Ciências da Saúde de Porto Alegre, Porto Alegre, RS, Brasil), Thiago Willers (Universidade Federal de Ciências da Saúde de Porto Alegre, Porto Alegre, RS, Brasil)

\section{BACKGROUND}

The evaluation that post-vaccine phenomena would be related to immune-mediated responses have caused these phenomena to be included in ASIA (Autoimmune Syndrome Induced by Adjuvants) syndrome.

\section{CASE REPORT}

An 25 years old woman, with jSLE. She was in good control of the disease, until she received the second dose of hepatitis B vaccine, with onset of pruritic scaly erythematous lesions on the face, scalp, neck and proximal region of arms (in photoexposed areas). There was no history of photoexposure and there was correct use of the sunscreen. We did not find any other systems disease. Laboratorially there was no change. The patient was treated with corticoid with good response. We chose not to do the third dose of the vaccine.

\section{CONCLUSION}

Adjuvants are substances that trigger immune response with innate and adaptive immune system activation to increase the response to that substance to which it is attached. It is very common to use in vaccines, currently the most used adjuvant is aluminum hydroxide. In addition to an immune system activation the adjuvant helps to preserve the main component allowing it to stay in the body longer, thus being more exposed to the immune system for a greater response.

In some cases after vaccination appear autoantibodies, nonspecific symptoms and even complete autoimmune disease.

More than $90 \%$ of patients present with joint manifestations such as arthralgia or arthritis, morning stiffness may occur, $>90 \%$ myalgia or muscle weakness (including with change in electromyography with pattern of myopathy), fever, nocturnal sweating, $>75 \%$ dry mouth and / or eyes (the minority with anti SSA or SSB present), 30-50\% Raynaud's phenomenon including changes suggestive of systemic sclerosis in capillaroscopy, 30-50\% may have neurological manifestations (including severe manifestations such as stroke, multiple sclerosis and amyotrophic lateral sclerosis), respiratory manifestations such as coughing, wheezing and dyspnoea may occur mainly in patients with previous asthma, abdominal pain, dysphagia and tachycardia can be found, urinary symptoms, generalized lymphadenopathy, 30-40\% livedo reticular, 20-40\% ill-defined rashes.

There is no strong scientific evidence but several case reports describing the development of rheumatic diseases, including SLE, following vaccination for HBV. 
Since our patient had a clear vaccine exposure it is important to think about the ASIA syndrome to explain reactivation of skin disease. It is important to remember that even with the described cases there is no contraindication in the literature of vaccination with attenuated virus in controlled SLE. 